\title{
Different Types of an Energy Efficient Multicast Routing Protocols for QOS in Wireless Communication
}

\author{
M Pushpalatha, M.Srinivasan, P Ramadevi
}

\begin{abstract}
This research paper proposes the "mobile ad hoc networks (MANETs) need aid autonomously self-organized networks without framework backing". For a "mobile ad hoc network, nodes move arbitrarily"; consequently that network might background fast also random topology changes.In view nodes previously, a MANET regularly have set transmission ranges, a percentage node can't correspond specifically with one another. Hence, routing path in mobile networks possibly hold numerous hops, each hub to mobile networks need the obligation on go about as a switch. This paper is an review from research work on "routing protocol for MANET, Mobile Ad Hoc Network" has as of late increased a ton of fame among computer researchers and specialists. "A MANET is an infrastructure less network" with a lot of dynamic, versatile and self-arranging hubs. Intrigue and utilization of remote versatile network have been becoming in the course of the most recent couple of years.MANETs to have a productive multicast directing and a Quality of Service (QoS) component.Multicast for Ad hoc Network with Hybrid Swarm Intelligence convention depends on swarm insight based optimization technique.
\end{abstract}

Keywords: Uni casting, Multicasting, DVMRP, Reactive and proactive protocol, AODV, MANET, QoS.

\section{INTRODUCTION}

The previous decade has encountered an exponential development in the utilization of wireless networks since they empower versatility - a trademark quality that isolates them from wired networks. This is the aftereffect of a wide range of elements including the accessibility of a collection of portable devices active research work for MANETs may be carrying with respect to principally in the fields of medium access control (MAC)[1] routing, asset management, force control, and security. Due to the vitality of directing conventions clinched alongside element multi jump networks, a considerable measure of MANET routing protocol have been suggested in the A couple a considerable length of time. Recognizing those uncommon properties from MANET at speculation around whatever routing protocol, by and large those taking after properties would expected, In spite of all of the could not a chance to be could reasonably be expected should incorporate single solution [2].
1. A routing protocol for MANET should be distributed in manner in order to increase itsreliability.

2. A routing protocol must be designed considering unidirectional links because wireless medium may cause a wireless link to be opened in unidirection only due to physicalfactors.

3. The routing protocol should bepower-efficient.

4. The routing protocol should consider itssecurity.

5. A hybrid routing protocol should be much more reactive than proactive to avoidoverhead.

6. A routing protocol should be aware of Quality of Service(QoS).

A network can be with or without a fixed infrastructure that empowers wireless mobile units to speak with one another. Examplea cellular network, base stations (BSs) as shown

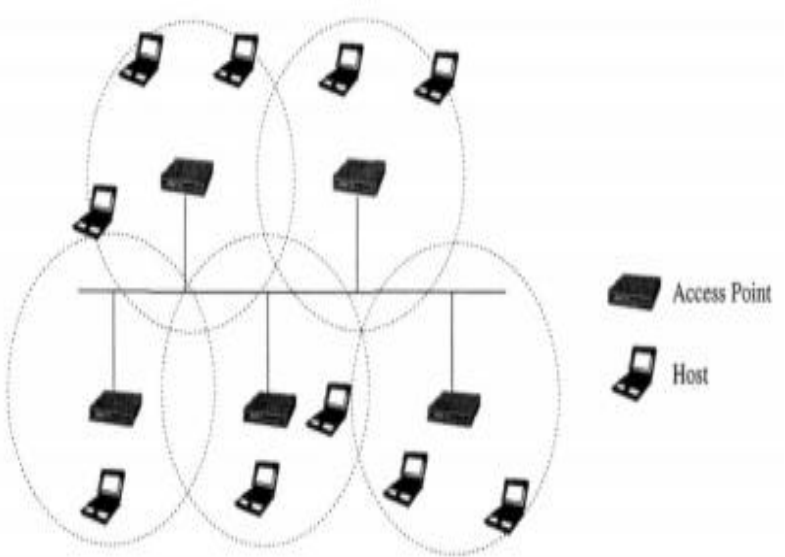

Figure.1. Infra-structured based network.

While in an infra-structure less network, there is no fixed infrastructure set up (i.e., there is no fixed BSs or routers). All the mobile nodes go about as both host and routers. Such a network is usually known as Mobile Ad hoc Networks (MANETs) and is delineated in figure 1.2[3]

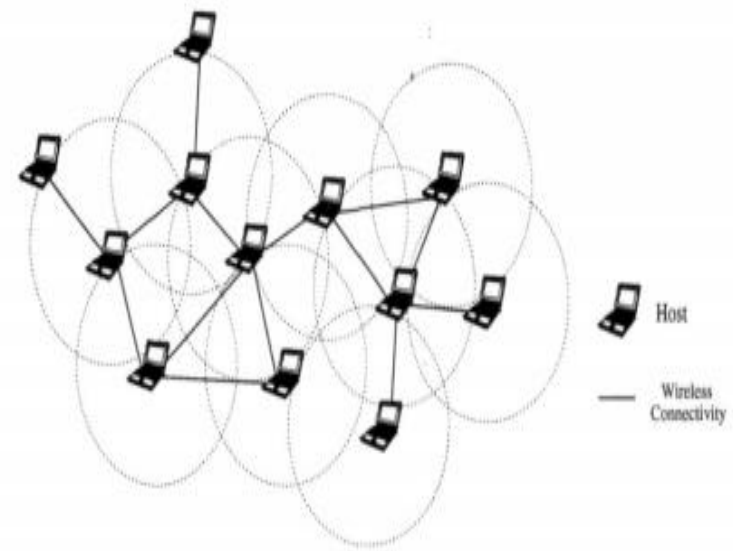

Figure.1.2. Infra-structure less based network

M.Pushpalatha, Research Scholar, Dept. Of ECE, SSSUTMS Sehore,Bhopal,Madhya Pradesh, India.

Dr.M.Srinivasan, Associate Professor, Dept. of ECE in SSSUTMS Sehore, Bhopal, Madhya Pradesh, India

Dr.P.Ramadevi, Associate Professor, Dept. of ECE, Vardaman College of Engineering, Hyderabad, India 


\section{RELATED WORK}

Various types of routing protocols for MANET, it has two types

\section{i. Proactive}

ii. Reactive

Proactive:"It is always learn the topology of the network by exchange topological data with the network nodes".Thus, when there is a have to a route to a destination, such route majority of the data is accessible instantly. Though the network topology progressions excessively frequently, the cost of maintain the network may be high".

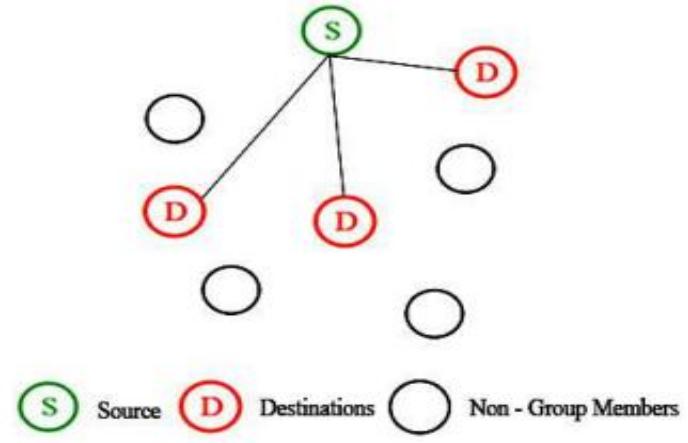

Figure.2. Different multicast routing protocols

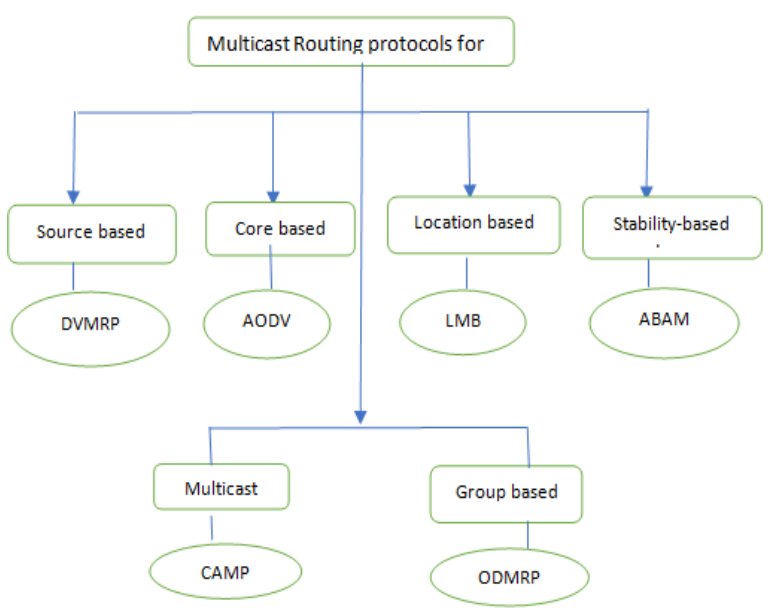

Figure.3. Types of existing ad hoc multicast routing techniques

\subsection{DVMRP with wireless Extension}

The "DVMRP with wireless extension distance vector multicast routing protocol" [4]. It is initially planned for wired network. The extension wasaccessible in [5] to allow DVMRP to task more well in a mobile ad hoc network. These extensions would leaf node identification element grafting/pruning Furthermore bundle duplication weigh. DVMRP administers wellspring based multicast conveyance trees. The hotspot based tree may be made by initially flooding the entirety network with the multicast traffic. Dynamic grafting/pruning is an additional development which serves in speeding dependent upon multicast tree reconfiguration the point when DVMRP will be utilized within mobile specially appointed system. In the first DVMRP, best changing grafting may be Gave with permit another part with rapidly join those multicast assembly. For dynamic grafting/pruning, when a nodes detects that those briefest way once again of the multicast sourball hub need changed (for example, At those multicast movement may be accepted starting with an alternate upstream node to span more extended over those edge duration)it will send a false message to the current upstream node and a graft message to the new upstream node. With this method, DVMRP multicast tree adapts faster network mobility.

\subsection{AODV Multicast}

Ad hoc on demand distance vector (AODV) [6] at first helps unicast also consequently cover multicast routingadditionally. It is an on-demand protocol in view of those end arrangement number idea presented over DSDV [7]. Arrangement numbers would used to determine the new routing data so that existing majority of the data could be promptly distinguished and more disposed of. These succession numbers would updatedjust by authorized nodes. For unicast routing protocol, just those end node will be permitted on upgrade those succession number of its own routing data (except conceivably when the route is broken). Previously, multicast routing protocol, a node is chosen with produce furthermore overhaul that multicast aggregation succession number. This node may be known as that multicast group leader. AODV multicast protocol makes also administers an absolute imparted tree for every multicast group. Each multicast one assembly needs an aggregation arrangement amount. This aggregation arrangement amount is occasionally incremented and more transmitted for the network by utilizing multicast group leader.Those to begin with node requesting a route will a specific multicast group naturally gets to be the pioneer of that multicast group. Therefore, those multicast tree is rapidly based at that point the ensuing nodes will join the group and the new part node will select the best on-tree node to unite. This Choice is in light of the new aggregation grouping amount and the jump separation will in turn to another group.

\subsection{Multicast Mesh: Camp}

(CAMP) [8] Makes a multicast network for each multicast group. Person or All the more center nodes are doled out with perform join operations in this way that flooding may be never again necessary. A multicast mesh can make made by utilizing both sender and receiver nodes which joins those multicast group. With join a multicast group, a node initial checks if any about its neighbors need aid recently parts of the multicast network. Whether true, it just necessities to publish its enrollment demand with its neighboring nodes. However, whether there are no network parts "around its neighbors, those join message will make sent on a standout amongst the center nodes.

\subsection{Group based: ODMRP}

ODMRP [9] is a flooding-based multicast routing protocol for mobile specially appointed networks. Dissimilar to the immaculate flooding scheme, information is not overflowed all around the organize previously, ODMRP. Instead, information will be overflowed just all around the forwarding group, which is ceaselessly administered by occasional flooding of control messages. Those sending group, which might have been to begin with "FGMP (Forwarding group Multicast Protocol") [10], will be a situated about especially ad hoc nodes extraordinarily picked will forward multicast movement for a specific multicast group.

The arrangement and support for this sending group confirms that all sending group nodes 
might give no less than particular case way starting with the multicast sender will at receivers. With secure and administer such sending group, ODMRP relies on the emulating operations:

a. Join Table broadcast by multicast receivers

b. Multicast sender advertisement

At a multicast sender need information should send, it begins the "periodic broadcast of JOIN-REQUEST messages". These "JOIN-REQUEST messages" are overflowed for those versatile specially appointed organize. Every node, upon getting that JOIN- appeal message, will upgrade its unicast directing table with that address of the hub starting with which that JOIN- solicitation message will be received.
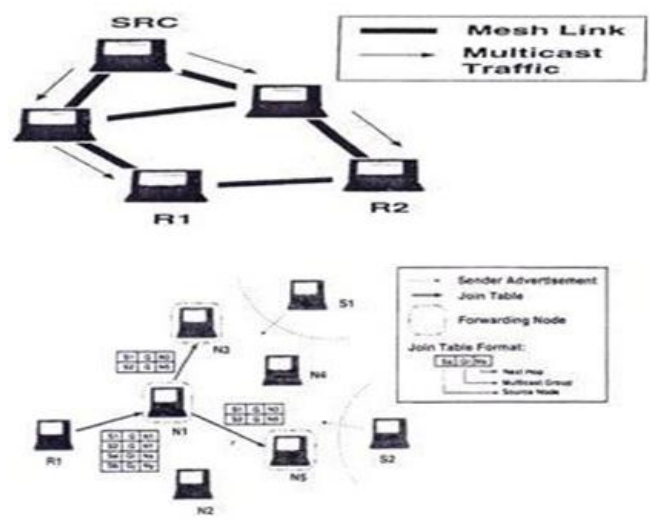

Figure.4. Multicast Receiver

Receivers those "JOIN-REQUEST message, it will redesign its part table for those location of the multicast sender also periodically broad cast JOIN-TABLE messages". "The JOIN- table message holds the rundown about every one multicast senders referred to that collector and the next-hop nodes towards the individual's multicast senders". Those next-hop data is promptly accessible from those unicast directing table. Best the node recorded as those following jump in the "JOIN-TABLE message" will transform those "JOIN-TABLE message". These hubs will turned sending group nodes Furthermore make those new JOIN-TABLE with the next-node majority of the data starting with its own message reserve. Those recently made "JOIN-TABLE will make show further". "JOIN-TABLE data" will a chance to be propagated go to all multicast senders also at nodes along the route starting with each recipient with every sender will be incorporated in the sending group, Likewise fig. 4 toward Hosting at these occasional messages, the sending gathering will make continually revived. A timeout component is that point used to uproot stale sending aggregation hubs. To addition; different ways are for the most part accessible through sending gathering thereabouts that when a connection may be broken, information packets are still sent along these exchange ways.

\subsection{Location Based Multicast: LBM}

LBM [8] utilizing area in formation will appropriate multicast traffic of the end multicast group, which will be likewise characterized In light of area. Dissimilar to the IP multicast model, LBM proposes a system that employments those area for middle of the road hubs should keep those flooding degree. This will be illustrated over figures (a) and more (b). In this method, that source node computes the sending zone that includes the multicast region and the extra area amidst that hotspot node and the multicast locale. For simplicity, LBM utilization a rectangular shape will define the region, likewise demonstrated over fig. A. The sending zone detail will be appended to every multicast packet furthermore of the multicast region determination. Main that node inside the sending zone will rebroadcast the packet.

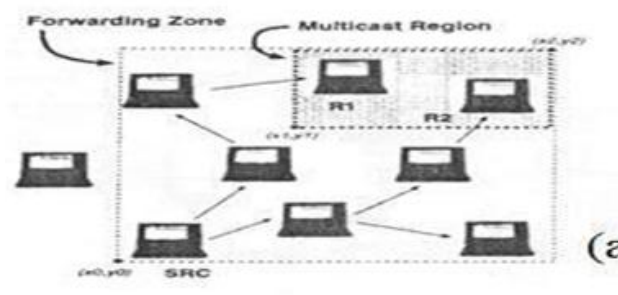

(a)

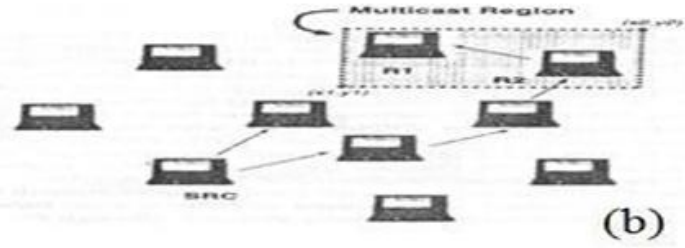

Figure.5. Location based Multicast

\section{PROACTIVE DSDV ROUTING PROTOCOL}

Dynamic destination sequence distance vector routing protocol (DSDV) [6] will be formed on the premise for Bellman-Ford routing [12] algorithm for a portion adjustments. In this routing protocol, every mobile node in the organize keeps a routing table. Every of the routing table holds the rundown from claiming the sum accessible destinations and the amount of jumps on every. Every table attempt may be labeled with an arrangement number, which may be originated by those end nodes. Periodic transmissions for updates of the routing tables help looking after that topology majority of the data of the system. Whether there may be at whatever new significant change to the routing information, those updates needs aid transmitted instantly.

So, those routing data updates could whichever make periodic alternately event- determined. DSDV protocol requires every mobile node in the organize will promote its own routing table to its current neighbors. That promotion will be carried out whichever television alternately by multicasting. Toward the advertisements, those neighboring node could think something like any progress that need struck them in the network because of the developments about nodes. Those routing updates might a chance to be sent on two ways: one will be called a "full dump" furthermore an additional is "incremental. " in the event from claiming full dump, the whole routing table is sent of the neighbors, the place likewise in the event that from incremental update, best those sections that require progressions are sent.

\section{REACTIVE ROUTING PROTOCOLS}

Dynamic Source Routing (DSR) [13] allows nodes in the MANET on rapidly uncover a source route over numerous system jumps to at whatever end. In this protocol, the mobile node would needed on look after course caches alternately the referred to routes. The route reserve may be updated at any new route may be referred to for a specific entrance in the route cache. Routing in DSR is done utilizing two phases: Route 
finding also route maintenance. At a source node needs with send a bundle should an destination, it main consults its route reserve will focus if it generally knows regarding any route of the end or not. Whether at that point there will be a passage to that destination, the source utilization that with send those bundle. Though not, it initiates a course a show. This request incorporates those end address, source address, furthermore an interesting distinguished. Every intermediate node checks if it knows around the end alternately not. Assuming that that middle of the node doesn't think regarding the destination, it once more advances the packet also inevitably this achieves destination

\section{Ad Hoc On-Demand Distance Vector Routing(AODV)}

AODV [14] will be essentially a change of DSDV. But, AODV may be a reactive protocol As opposed to proactive. It minimizes that number for telecasts by making routes dependent upon demand, which will be not the case of evidence for DSDV. At any source node needs to send a packet to destination, it telecasts route request (RREQ) packet. The neighboring nodes thus show that packet on their neighbors and the methodology proceeds until the packet achieves the end. Throughout that procedure of sending that course request, intermediate nodes record the address of the neighbor from which those initial copy of the show packet is accepted. This record will be stored on their route tables, which helps to secure opposite way. In extra copy of the same RREQ is later received, these packets are disposed and those relay may be sent utilizing those reverse path. To course maintenance, when a hotspot node moves, it might re- launch a course revelation procedure. In any intermediate node moves inside a specific route, those neighbor of the drifted node might recognize the connection failures and sends failure node notice to its upstream neighbor. This procedure continued until that failure notice achieves the source node. In view of those gained information, those source could choose to be re-initiate those route disclosure stage.

\section{HYBRID ROUTING PROTOCOLS}

\section{Adaptive distance vector routing (ADV)}

ADV [15] routing protocol is a distance-vector routing method that exhibits some on-demand features Toward changing those recurrence and the extent of routing updates because of the response to the network load and portability designs. ADV utilizes an adaptive system that relieves that impact about occasional transmissions of the routing updates, which fundamentally depends on the network load and versatility states. To decrease the size for routing updates, ADV advertises and administers routes to the dynamic receivers. Just a node will be viewed as active that it may be the receiver for whatever at present active association. There will be a receiver flag in the routing entry, which keeps those data something like the status of a recipient by not bothering about active or inactive. Here send data, a source node telecasts network-wide an in itconnection control packet. Here all the other nodes turn on the corresponding recipient flag over their routing tables and begin advertising those routes of the collector in future updates. When the end node gets in to it-connection packet, it responds to it by telecasting a receiver-alert packet and gets to be dynamic. To close a connection, the source node telecasts network-wide a limit association control packet, demonstrating that that association will be closed. Whether those end node does not need any extra dynamic connection,

\section{CONCLUSION}

This paper presents a number of routing protocols for MANET for improvement of Quality of service (QoS) that are comprehensively sorted as proactive and sensitive. Proactive routing routing protocol tend for providing lower latency that of the on-demand protocols, a result they attempt to maintain routes to all nodes in network constantly. But the drawback to such protocols is those unreasonable routing overhead transmitted which will be occasional in nature without a great consideration for the network mobility or load. On the different hand, if sensitive protocols find routes just when they need might even now produce an enormous amount of traffic at those network changes frequently. Relying upon the amount of network traffic and number of flows, those routing protocol Might be picked. Here there will be congestion in the network because of huge traffic, in general case; a sensitive protocol will be preferable. A few times the span of the system may a chance to be a major significant point. To example, AODV, DSR, OLSR are a few of the protocol suitable for moderately smaller networks.

\section{REFERENCES}

1. Sethi,S\&Udgata, SK 2010, 'Scalable Cluster Based Ad hoc Ondemand Distance Vector Routing Protocol for MANET', In Proceedings of IEEE International Conference on Communication and Sensor Networks, pp. 1-6.

2. Venugopalan, R \& Daniel, M, 2002, 'SRL: Providing a Bidirectional Abstraction for Unidirectional Ad Hoc Networks', Online technical report, url: http://www.cs.cornell.edu/amasv/SRL/SRL(ToN).pdf.

3. Usha, M, Jayabharathi, S \& Banu, RW 2011, 'RE-AODV: An Enhanced Routing Algorithm for QoS Support in Wireless Ad-Hoc Sensor Networks', In Proceedings of the IEEE International Conference on Recent Trends in Information Technology, pp. 567 571 .

4. S. Deering, C. Partridge, and D. Waitzman, "Distance vector multicast routing protocol," RFC, Tech. Rep.,1988.

5. Y.Yi, “On demandmulticastroutingprotocol(odmrp)foradhocnetworks," draftyi-manet-odmrp-00, 2003.

6. C.Perkins,E.Belding-Royer,andS.Das, "Adhocon demanddistanceVector (aodv) routing," Tech. Rep., 2003.

7. C. E. Perkins and P. Bhagwat, "Highly dynamic destinationsequenced distance-vector routing (dsdv) for mobile computers," in ACM SIG- COMM computer communication review, vol. 24, no. 4 ACM, 1994, pp. 234-244.

8. J. Garcia-Luna-Aceves and E. L. Madruga, "The core-assisted mesh protocol," Selected Areas in Communications, IEEE Journal on, vol. 17 ,no. 8, pp. $1380-1394,1999$.

9. M. Gerla, C.-C. Chiang, and L. Zhang, "Tree multicast strategies in mo- bile, multihop wireless networks," Mobile Networks and Applications, vol. 4, no. 3, pp. 193-207,1999.

10. C.-C. Chiang, M. Gerla, and L. Zhang, "Forwarding group multicast protocol (fgmp) for multihop, mobile wireless networks," Cluster Com- puting, vol. 1, no. 2, pp. 187-196,1998.

11. Y.-B. Ko and N. H. Vaidya, "Location-based multicast inmobileadhocnetworks," Texas A \& M University, College Station, TX, 1998.

12. Cheng C, Riley R, Kumar SPR, Garcia-Luna-Aceves JJ (1989) A Loop- Free Extended Bellman-Ford Routing Protocol Without Bouncing Effect. ACM 
SIGCOMM Computer Communications Review, Volume 19 , Issue 4:224-236.

13. Broch J, Johnson DB, Maltz DA (1999) The Dynamic Source Routing Protocol for Mobile Ad Hoc Networks. IETF Draft, October, 1999, available at http://tools.ietf.org/id/draft-ietf-manetdsr-03.txt. Accessed 21 February2008

14. Perkins CE, Royer EM, Chakeres ID (2003) Ad hoc On-Demand Distance Vector (AODV) Routing. IETF Draft, October, 2003, available at http://tools.ietf.org/html/draft-perkins-manet-aodvbis00. Accessed 21 February2008.

15. Boppana RV, Konduru SP (2001) An Adaptive Distance Vector Routing Algorithm for Mobile, Ad Hoc Networks. Proceedings of IEEE INFOCOM2001:1753-1762

\section{AUTHOR'S PROFILES}

M. Pushpalatha She got B.Tech degree from GNITS Engineering college in ECE branch from Hyderabad. she got M.Tech degree from MIST Engineering College with DECS from Hyderabad. Sheis doing Ph.D. from SSSUTMS Sehore,Bhopal, Madhya Pradesh in the department of ECE. she is the permanent member of ISTE ,IETE and AIENG.she has 12 years of teaching experience in engineering field. she attended many workshops related to wirelesscommunication andin other domain. she published 13 papers in various internationaljournals attended many conferences. she has very much interest to do research on Wireless Communication,VLSITechnology,embeddedsystems and Digital Electronics.

Dr. M. Srinivasan working as an ASSOCIATE PROFESSOR in SSSUTMS Shore, Bhopal, Madhya Pradesh. He has 18 years of Teaching Experience; he published 14 papers in various journals. He has very much interest to do research on VLSI Technology and Design, communication systems and Digital Electronics and Wireless Communication.

Dr. P. Ramadevi She has completed herPh.D. in the area of WIRELESS NETWORK from JNTU Hyderabad, and Prior to this she has completed master's degree ME in Applied Electronics fromUniversity of Madras, she published total 15 papers in national and international journals and conferences, and she is a member of MISTE and FIETE, she has total 17 years of experience in teaching field she attended and organized seminars, workshops and FDP programs. She is very much interest for the research in the field of wireless communication, digital electronics, Presently working as an ASSOCIATE PROFESSOR in jntuh affiliated college. 